\title{
Evaluation of Multi-agent Architecture for Structural Damage Detection and Location
}

\author{
https://doi.org/10.3991/ijoe.v13i06.7184 \\ Andres F. Quintero-Parra \\ Universidad Simón Bolivar, Barranquilla, Colombia \\ aquintero30@unisimonbolivar.edu.co \\ Jhonatan Camacho-Navarro \\ Universitaria de Investigación y Desarrollo, Bucaramanga, Colombia \\ jcamacho2@udi.edu.co \\ Marco Flórez \\ Universitaria de Investigación y Desarrollo, Bucaramanga, Colombia \\ marco.florez@udi.edu.co \\ J.L. Vázquez-González \\ Universidad de las Américas Puebla UDLAP, Cholula Puebla, México \\ josel.vazquez@udlap.mx
}

\begin{abstract}
In this paper the results of using a Multi-agent system (MAS) for Structural Health Monitoring (SHM) are detailed. A study between different MAS architectures reported in literature is presented in order to select and adapt the most adequate one for SHM tasks. Requirements are established according to recent solutions, where main parameters are type and number of sensors and communication protocols, among others. MAS technique uses several intelligent agents, that are algorithms able to act in a reactive or active way. Their action depends on surrounding environment or collected data. These agents can work in a decentralized way, searching the fulfillment of an individual goal or they can work with another system to achieve a common goal. Decision is based on their internal state (beliefs, goals and commitments). MAS' effectiveness depends on the interconnection between the agents. Type of agents is defined according to its communication method and protocol, common and individual goals, among others. Decentralization and versatility are two important characteristics of MAS technique useful to solve SHM problem. This is one of the main motivations to consider this technique to be a good approach for the studied problem. A benchmark numerical model, which consists of a metallic framework, was used to validate and demonstrate the feasibility of the selected architecture for SHM.
\end{abstract}

Keywords-Artificial Intelligence, Intelligent Agent, Multiagent Architecture, Structural Health Monitoring 


\section{Introduction}

Structural health monitoring is the common name for the structural damage detection problem. Many solutions have been proposed, some of them based on simple observation executed by the railroad workers on the XIX century. Visual recognition techniques are used when there is no available technology to accomplish a better study. The computational advance in the last thirty years, has proven to be a determining factor in the uprising on the number and quality of SHM techniques [1]. Some SHM techniques, like Wavelet Transform deals with the identification of discontinuities on acceleration records to detect fault's initial point [2]. Another use of Wavelet is finding changes in the flexibility matrix to detect the existence of any kind of damage [3]. There are some specialized works that deals with the quality of the analyzed signals, in order to perform a better damage estimation. For example, [4] proposes a de-noising method for the sensor signals based on the variance of the whole data compilation. There are other techniques implemented in places and situations where SHM is not easily performable. One example is shown in [5], where its author uses genetic algorithms to improve the approximation of the DA (Delay Attenuation) model that is used to estimate the structure features. There are many other techniques designed to perform damage determination and damage geometrical location (levels 1 and 2 of damage evaluation), but they can be classified, according to [6] in two types: Techniques based in modal form detection and Techniques based in parameter extraction. Most of the current implementations of SHM are based on Expert Systems [7]. A case is that reported in [8] named SHIELD (Structural Health with Integrated Life Determination) that uses wireless nodes and a central system to estimate accumulated fatigue of an earthmoving machine. However, according to [9] a centralized system is slower and less adaptive than a decentralized one. Based on this, an appropriate solution for SHM is one based on distributed systems, because relevant information of the monitored structure can be contained in geographically distributed points. Thus, because MAS are distributed systems with an architecture adjusted for the specific problem, a specific architecture could be used for SHM, and consequently a study of existing multi-agent architectures is necessary in order to select the most adequate and adapt it for a specific structural model. Taking advantage of Multi-agent characteristics, this paper investigates the current solutions of the SHM problem and the existing multi-agent architectures, in order to propose a solution to the SHM problem based on MAS approach, due to the similarities between monitoring problems and SHM. Finally, a Benchmark numerical model is used to verify the adapted and selected architecture.

\section{Structural Health Monitoring (SHM)}

SHM systems can be classified in 4 levels according to their functionality in the damage determination process: 
Level 1: Structural damage detection

Level 2: Level 1 plus damage location

Level 3: Level 2 plus damage quantification

Level 4: Level 3 plus useful life estimation also called prognosis [1].

There are techniques that use the modal forms to acquire free vibration registers to solve SHM levels. The modal forms are the answers of a structure due to inputs vibrating at a close frequency range to the natural frequency of the structure [9]. The dynamic properties of a structure can be found through interpretative mathematical analysis on the modal forms [6]. These registers represent the natural frequencies of the structure. Some of the techniques based on modal forms are ERA (Eigensystem Realization Algorithm) and ERA/DC (Eigensystem Realization Algorithm with Data Correlation). There are other techniques that use the free vibration registers to obtain the modal forms of a structure. For example, NExT(Natural Excitation Technique) is one of these techniques and can be used to determine bridge's modal forms [1].

Other approaches documented in literature are based on parametric identification techniques, which use mathematical processes to extract information about the structures lacking the use of modal forms. For instance, FDD [10] and shear beam model [11] obtain parametric data of a Benchmark without statistical models. Likewise, Hidden Markov Models can be used as a sequence classifier to obtain structural parameters [12] or to estimate structural lifetime regardless of the damage estimation method used [13]. Also, Bayesian networks can be used for structural damage location [14]

One of the commonly used techniques for damage detection is model updating, where a mathematical model of the structure is updated with every measure taken and then compared with the model of the healthy structure. Another technique is to use the structure's modal forms to determine if there is any change between the measured and the original ones. However these techniques use a large amount of structural information taken from a sensor network where everyone should be operating correctly. When a sensor fails these systems can't execute their analysis and, therefore, can't present a reliable diagnosis creating a system robustness problem undesired by engineers [15].

Current monitoring systems use hybrid architectures to take advantage of the distributed external data, and to perform data analysis, take the best course of action to detect failures as soon as possible [2]. For instance, in [16] a MAS based on a four levels hierarchical architecture was used for SHM that can be used as reference point to evaluate SHM strategy to be proposed in this research project. The main reason is that according to reviewed literature this is the only SHM strategy found using MAS.

\section{Multi-Agent Architectures For SHM}

Intelligent agents are algorithms capable of active and reactive behavior, based on BDI (Belief-Desire-Interaction) model. Figure 1 shows an agent composition and its relationship with its surrounding environment. An agent percepts its surrounding 
environment through sensors, makes data processing, data analysis, decision making process and the uses its actuators to interact with its environment. They can be grouped into a structure known as Multi-agent architecture [17] [18] [19].

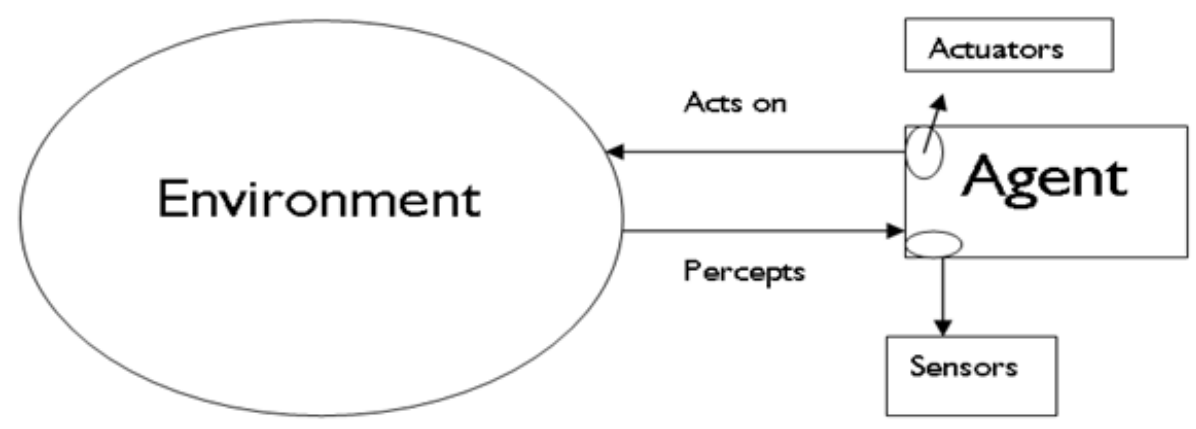

Fig. 1. Agent's action and reaction model

Most applications of MAS are focused on solving distributed problems. Data distribution can be done more efficiently by a multi-agent architecture than a sensor network [20]. Concurrent task execution is a multi-agent architecture characteristic used to improve efficiency in distributed systems [21]. In [22] it is explained the use of a role-based multi-agent architecture to obtain a general vision of a distributed system. Additionally, in [23] it is presented MACRO (Multi-agent Architecture for Coordinated Responsive Observations) as a solution to SEAMONSTER (South East Alaska Monitoring Network for Science, Telecommunications, Education and Research). MACRO is based on a hierarchical organization. Other example is presented in [24], where a work based on Situation Calculus that uses intelligent agents for dynamic environment monitoring such as battlefields or buildings is described. One of the common applications of multi-agent architectures is robot control, and the rolebased architecture is a common choice for this problem [25] [26] [27].

Although MAS have lot of applications in distributed problems, there are few SHM works. The following works present some interesting approaches to the problem: [28] proposes a technique to use mobile agents, such as robots, to detect structural faults and provide a minimal repairing service. This mobile agent should be able to fit into a MAS used for damage detection. [16] Designs a multi-agent architecture focused on SHM. Types, ontological function, goals, communication routes of each agent are described. Also, the integration of each agent into SHM is explained. [29] Proposes a mobile agent to be used in SHM. Every sensor is checked periodically by the agent. Based on the collected data, structural health can be determined by the agent. [30] Evaluates a multi-agent architecture used for SHM. Interactions, types and locations of each agent are specified. 


\section{$4 \quad$ Architecture's Adaptation}

Table 1 shows a comparison of the MA architectures based on their flexibility, relation to SHM problem and number of agents. Flexibility is related to how agents can change their roles in order to perform better or how they can move through the architecture. The second parameter refers to the number of different agents that can be supported by the architecture.

Table 1. Multi Agent Architectures

\begin{tabular}{|l|c|c|c|}
\hline \multicolumn{1}{|c|}{ Architecture } & Flexibility & Number of Agents & Type of Architecture \\
\hline ARCHON & Low & Medium & Hybrid \\
\hline CARBA & Medium & Variable & Reactive \\
\hline ORG & Medium & Variable & Logic \\
\hline MAGMA & High & Low & Logic \\
\hline Proposed by Yuan & Low & Variable & Hybrid \\
\hline
\end{tabular}

Considering SHM features, logic and reactive architectures are not adequate to achieve SHM levels, so only the hybrid architectures can present a correct diagnosis based on inference and data obtained from external sources.

According to its functionality, there are 7 types of agents: Structural Monitoring Agent (SMA), Data Handler Agents (DHA), SHM Transport Agent (STA), Translation Agents (TA), User Interface Agent (UIA), Central Collaboration Agent (CCA) and Global Data Merger. The SMA transforms the data from the sensors into messages understandable by every other agent. DHA manage the information flow through the subsystem. STA allows for communicating with other STA agents from other subsystems to share information about their position on the structure. TA determines which data will be needed by the STA and suggests to the DHA an itinerary. The UIA presents the results to the user and interprets the input commands. The CCA uses the data obtained from the data merger and its communication with the database to obtain a global structural diagnosis. Finally, the Global Data Merger uses data from every STA to create an array that can be used by the CCA to present a diagnosis.

In Figure 2 the adapted architecture is depicted, which details how the different agent types are specified. 


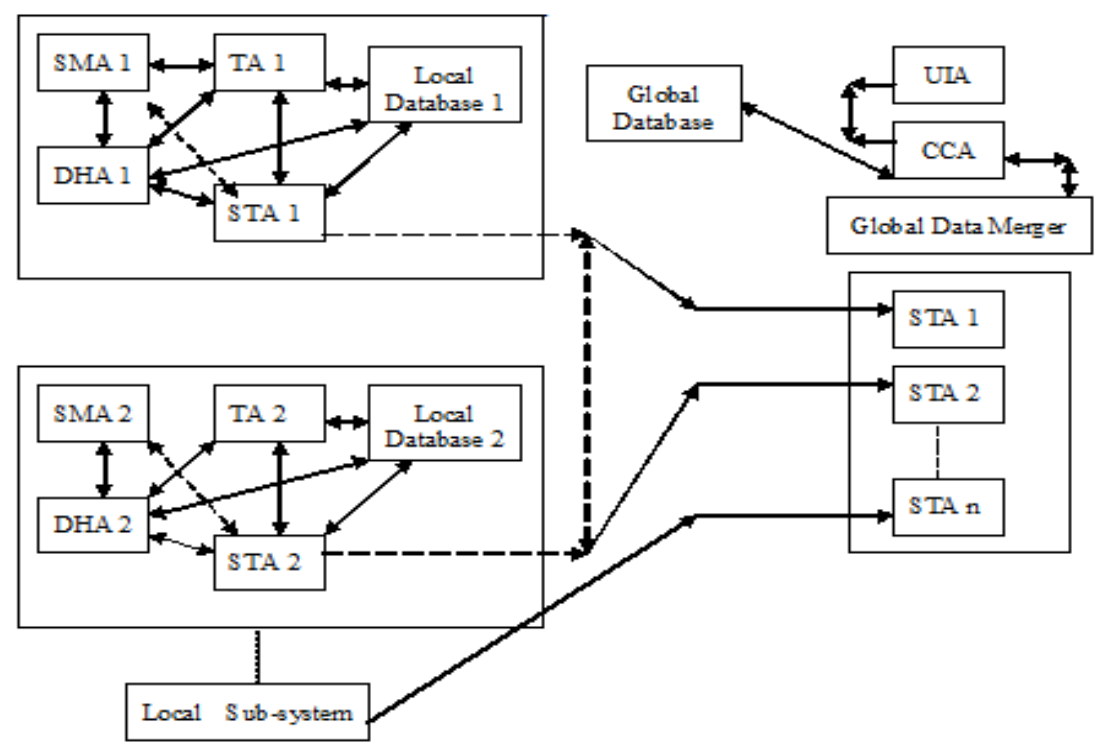

Fig. 2. Adapted Multi Agent Architecture

\section{$5 \quad$ Numerical Case}

To validate, numerically, the architecture will be tested with data obtained from a Benchmark proposed by the ASCE available online at http://mase.wustl.edu/ wusceel/quakeby December 2009 (Figure 3).

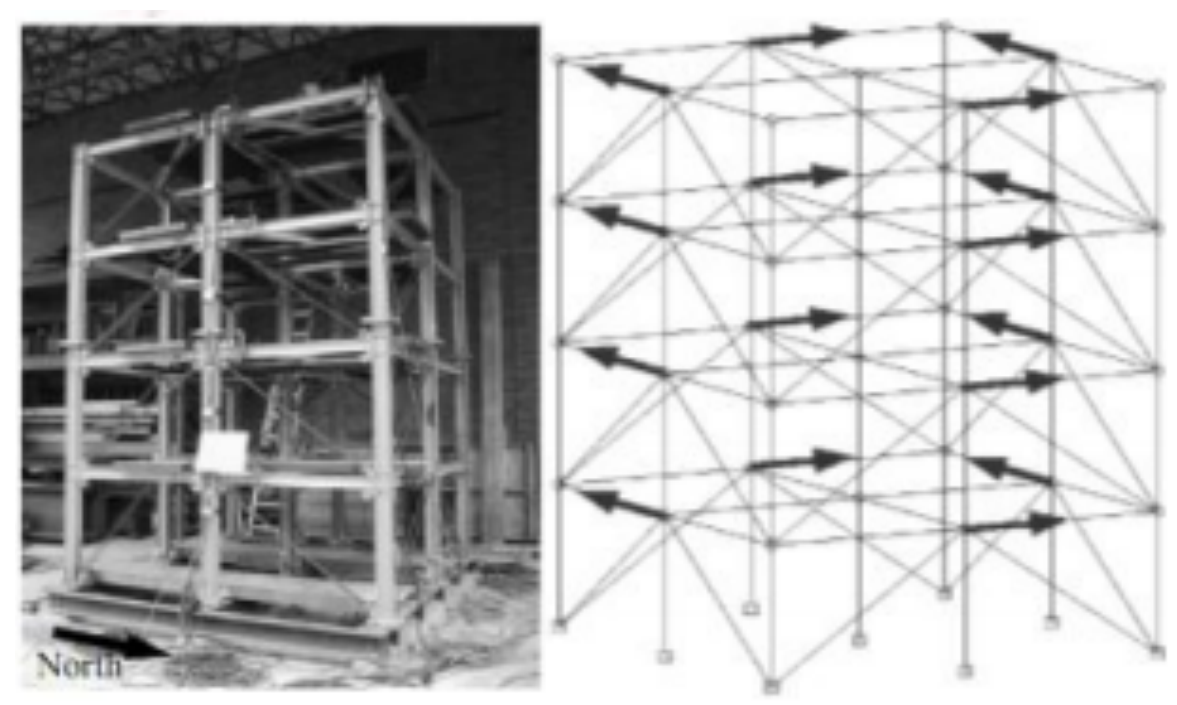

Fig. 3. Benchmark structure 
To validate the system's functionality, numerical data of the metallic frame is used to differentiate between healthy and damage states of the structure. Therefore the SMA agents will only deal with acceleration data and their output messages only deliver information in FIPA language. Damage cases consider different scenarios:

- $100 \%$ of stiffness change in only one element.

- Stiffness changes on more than one element in order to test system's adaptability.

- Variation of stiffness ranging from $10 \%$ to $100 \%$ advancing $10 \%$ at a time.

- Sensor and agent removing.

The system has this information on its local and global databases, so it has the capability to locate the specific case for every sensor. It must find the correct case in the global database to determine if a change has occurred.

\section{$6 \quad$ Results}

Every agent is programmed using JADE (Java Agent Development Framework). Local databases have Fourier and Wavelet transformations from acceleration data obtained from the structure numerical model. Fourier and Wavelet transform to obtain two kinds of data are managed for TAs agents. STAs can distinguish between Fourier and Wavelet coefficients to execute an estimation process and compare the results to the healthy structural data by means of a neural network, used by the CCA. The CCA search for data related with a healthy state of a specific sensor. It is achieved using the global database organized in a neural network, where one neuron is defined as a damage case.

With the aim to test the capability of finding the correct case in the global database, some scenarios consider STA "killing". Also, STAs were removed randomly to prove the system's awareness and its capability of damage detection without them. Local and global diagnosis obtained by each STA is summarized in Table 2.

Table 2. STA Diagnostics

\begin{tabular}{|c|c|c|}
\hline & Tag of damaged elements & Ocurrence \\
\hline STA 1 & $34-38-35-30$ & $100-99-99-99$ \\
\hline STA 2 & $34-38-30-35$ & $100-99-99-99$ \\
\hline STA 3 & $34-35-30-38$ & $100-99-99-99$ \\
\hline STA 4 & $34-38-30-35$ & $100-99-99-99$ \\
\hline STA global & $32-33-34-36$ & $120-120-120-120$ \\
\hline
\end{tabular}

Table 2 can be interpreted as damage probabilities as shown in Figure 4. 


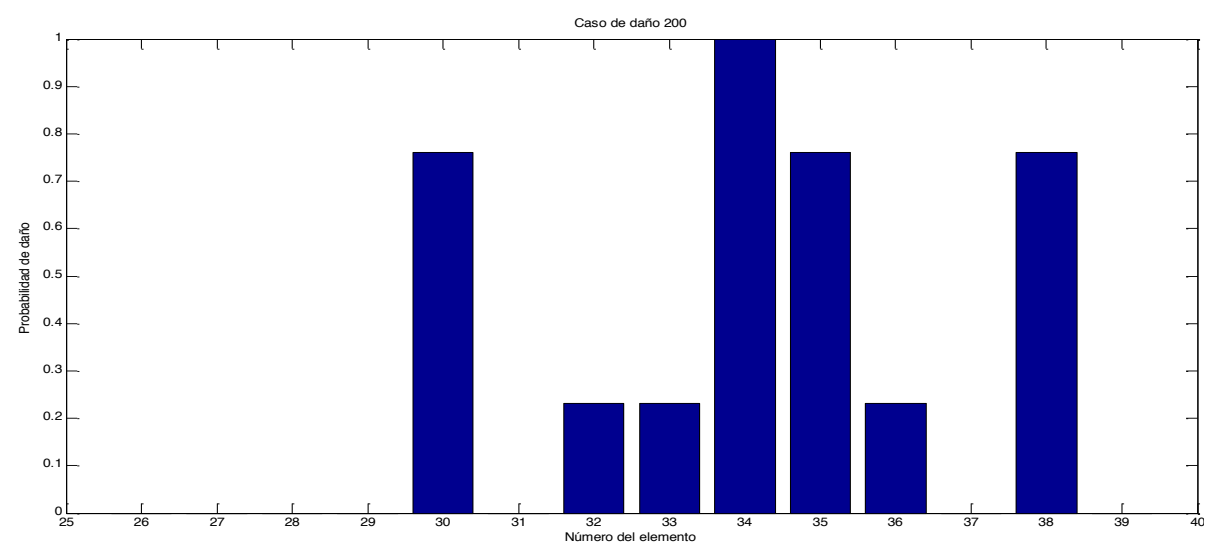

Fig. 4. Damage probabilities

\section{Conclusion}

The adaptation of a promising multi-agent architecture was presented as an alternative approach for SHM problem. The methodology minimizes latency in communications between local monitoring points and the central coordination agent. Thus, decentralized feature was incorporated to interpret effectively damage conditions. The architecture includes data fusion using a minimum number of sensors. The effectiveness of the algorithm was validated through a Benchmark with capability for processing acceleration records. As a future research it is recommended to use feature reduction techniques and complementary diagnostics tools.

\section{Acknowledgment}

Special thanks to Universidad Simon Bolivar, Universitaria de Investigación y Desarrollo and Universidad de las Américas Puebla UDLAP for their support on our investigation. Also Rodolfo Villamizar-Mejía and Juan Carlos García Ojeda advices were very useful to guide this investigation.

\section{References}

[1] C.R. Farrar and S. Doebling, "A Review of Structural Health Monitoring Literature : 19962001," Structural Health Monitoring, 2003, pp. 1996-2001.

[2] N.R. Jennings and W. College, "Applications of Intelligent Agents," Intelligent agents, 1996.

[3] W. Jacak, K. Pro, and S. Dreiseitl, "Conflict Management in an Intelligent Multiagent Robotic System," IEEE International Conference on Systems, Man, and Cybernetics, Nashville: 2000 , pp. 1793-1798. 
[4] H. Zhu, "A role-based architecture for intelligent agent systems," Proceedings of the IEEE Workshop on Distributed Intelligent Systems: Collective Intelligence and Its Applications, 2006.

[5] S.Z.H. Zaidi, S.S.R. Abidi, M. S., and Y.-N. Cheah, "ADMI: A Multi-Agent Architecture to Autonomously Generate Data Mining Services," International conference on intelligent systems, IEEE, 2004, pp. 273-279.

[6] P. Avitabile, "Experimental Modal Analysis ( A Simple Non-Mathematical Presentation )," Modal Analysis, 2004, pp. 1-15.

[7] H. Voos, M.A. Lopez-Carmona, M.-M. Iván, and J.R. Velasco, Multiagent Systems, InTeh, 2009.

[8] M. Scheutz and V. Andronache, The APOC Framework for the Comparison of Agent Architectures, Notre Dame: 2004.

[9] L. Kong and L. Xiao, "A Multi-Layered Control Architecture of Intelligent Agent," IEEE International Conference on Control and Automation, Guangzhou: 2007, pp. 1454-1458.

[10] B. Horling, R. Mailler, and V. Lesser, "A Case Study of Organizational Effects in a Distributed Sensor Network," IEEE/WIC/ACM International Conference on Intelligent Agent Technology, 2004, pp. 51-57.

[11] W.R. Otte, J.S. Kinnebrew, D.C. Schmidt, and G. Biswas, "A flexible infrastructure for distributed deployment in adaptive sensor webs," 2009 IEEE Aerospace conference, Mar. 2009, pp. 1-12.

[12] A. Kazandzhiev, I. Momtchev, L. Popova, and D. Shikalanov, "Distributed multi-agent based approaches," International Conference on Integration of Knowledge Intensive MultiAgent Systems, Waltham: Ieee, 2005, pp. 3-8. https://doi.org/10.1109/kimas.2005. 1427043

[13] S. Ibarra, C. Quintero, J. De La Rosa, and J. R. Castan, "An Approach based on New Coordination Mechanisms to Improve the Teamwork of Cooperative Intelligent Agents," 2006 Seventh Mexican International Conference on Computer Science, Sep. 2006, pp. 164-172. https://doi.org/10.1109/ENC.2006.5

[14] T. Fukuda, I. Takagawa, and Y. Hasegawa, "From intelligent robot to multi-agent robotic system self-organizing robotic systems," International Conference on Integration of Knowledge Intensive Multi-Agent Systems, Boston: 2003, pp. 413-417.

[15] X. Zhao, S. Yuan, Z. Yu, W. Ye, and J. Cao, "Designing strategy for multi-agent system based large structural health monitoring," Expert Systems with Applications, vol. 34, 2008, pp. 1154-1168. https://doi.org/10.1016/j.eswa.2006.12.022

[16] S.E. Lander, "Issues in multiagent design systems," IEEE Expert, vol. 12, Mar. 1997, pp. 18-26. https://doi.org/10.1109/64.585100

[17] M. Wooldridge, N.R. Jennings, and D. Kinny, "The Gaia methodology for agent-oriented analysis and design," Proceedings of the third annual conference on Autonomous Agents, 1999, pp. 69-76. https://doi.org/10.1145/301136.301165

[18] H.S. Nwana, D.T. Ndumu, L.C. Lee, B.T. Laboratories, and M. Heath, ZEUS: An Advanced Tool-Kit for Engineering Distributed Multi-Agent Systems, Ipswich, Suffolk: 2006.

[19] X. Zhao, S. Yuan, Z. Yu, W. Ye, and J. Cao, "Designing strategy for multi-agent system based large structural health monitoring," Expert Systems with Applications, vol. 34, 2008, pp. 1154-1168. https://doi.org/10.1016/j.eswa.2006.12.022

[20] S. Yuan, X. Lai, X. Zhao, X. Xu, and L. Zhang, "Distributed structural health monitoring system based on smart wireless sensor and multi-agent technology," Smart Materials and Structures, vol. 15, 2006, pp. 1-8. https://doi.org/10.1088/0964-1726/15/1/029 
[21] A.F. Quintero-Parra and R. Villamizar-Mejía, "Estado del arte en monitorización de salud estructural: un enfoque basado en agentes inteligentes," Ciencia e Ingeniería neogranadina, vol. 20, 2010, pp. 1-14

[22] F. Klügl, "A validation methodology for agent-based simulations," Proceedings of the 2008 ACM symposium on Applied computing, New York, New York, USA: ACM Press, 2008, p. 39. https://doi.org/10.1145/1363686.1363696

[23] R. Fuentes, J.J. Gómez-sanz, and J. Pavón, "Verification and Validation Techniques for Multi- Agent Systems," Upgrade, vol. 5, 2004, pp. 15-19.

[24] D.G. Fabio Bellifemine, Giovanni Caire, Developing Multi Agent Systems with JADE, John Wiley \& Sons, 2007. https://doi.org/10.1002/9780470058411

[25] J. Thangarajah and M. Winikoff, "Tool Support for Agent Development using the Prometheus Methodology," Fifth International Conference on Quality Software, Ieee, 2005, pp. 383-388.

[26] Juan Martin Caicedo, "Structural Health Monitoring of Flexible Civil Structures," Washington University, 2003.

[27] J. Wu, S. Yuan, S. Ji, G. Zhou, Y. Wang, and Z. Wang, "Multi-agent system design and evaluation for collaborative wireless sensor network in large structure health monitoring," Expert Systems with Applications, vol. 37, Mar. 2010, pp. 2028-2036 https://doi.org/10.1016/j.eswa.2009.06.098

[28] Hoschke, N., Lewis, C. J., Price, D. C., Scott, D. A., Edwards, G. C., Batten, A., "A SelfOrganizing Sensing System For Structural Health Management" Knowledge-Based Intelligent Information And Engineering Systems, Proceedings, Lecture Notes In Artificial Intelligence, 2006, Páginas: 349-357

[29] Taylor, Stuart G., Farinholt, Kevin M., Flynn, Eric B., Figueiredo, Eloi, Mascarenas, David L., Moro, Erik A., Park, Gyuhae, Todd, Michael D., Farrar, Charles R., "A MobileAgent-Based Wireless Sensing Network For Structural Monitoring Applications" Measurement Science \& Technology, Abril 2009. https://doi.org/10.1088/09570233/20/4/045201

[30] Zhou, Hengbao, Sun, Hongbing, Qiu, Lei, "An Evaluation on the Multi-Agent System Based Structural Health Monitoring For Large Scale Structures", Expert Systems with Applications, 2008, Páginas: 4900-4914

\section{Authors}

Andres F. Quintero-Parra is a Colombian master on electronics Engineer, who works as a teacher/researcher at Universidad Simon Bolivar in Barranquilla, Colombia. He works as an advisor for Apps.co initiative from Colombian TIC's minister.

J. Camacho-Navarro, is with Universitaria de Investigación y Desarrollo, Colombia.

M. Flórez is with Universitaria de Investigación y Desarrollo, Colombia.

J.L. Vázquez-González, is with Universidad de las Américas Puebla UDLAP.

Article submitted 10 May 2017. Published as resubmitted by the authors 15 June 2017. 